\title{
BREASTFEEDING IN RHEUMATOID ARTHRITIS IN ROMANIA
}

\author{
Anca Bobirca, Ioan Ancuta, Florin Bobirca, Cristina Tataru, Cristina Comsa, Carina Mihai, \\ Mihai Bojinca, Victor Stoica \\ Dr. Ion Cantacuzino Clinical Hospital, Carol Davila University of Medicine and Pharmacy, Bucharest
}

\begin{abstract}
Background. Rheumatoid arthritis (RA) is not a contraindication to pregnancy. It is only mandatory to observe several recommendations: planning pregnancy, adapting anti-rheumatic medication and monitoring disease activity every trimester. Postpartum, RA often relapses, therefore, breastfeeding can be exposed to anti-rheumatic drugs. Only Methotrexate, Leflunomide and Cyclophosphamide are fully contraindicated during lactation. TNF inhibitors are compatible with breastfeeding, while other biological therapies are not recommended, due to the lack of data.

Objectives. This study aimed to evaluate pregnancy outcomes among female patients with RA, who attended rheumatology clinics from different Romanian cities. Secondary objectives were disease course and anti-rheumatic medication post-partum, the occurrence of RA postpartum flares and the adherence to breastfeeding. Patients and methods. This is a pilot observational, multicentric study, performed between October 2012 and July 2016. We have analyzed 41 patients diagnosed with RA before conception and who had at least one pregnancy after diagnosis. We recorded the pregnancy outcome, and, regarding the postpartum period, the occurrence of disease relapse and the antirheumatic medication exposure during lactation.

Results. The 41 patients had 72 pregnancies, with 39 deliveries resulting in 42 healthy infants; 30 patients chose to breastfeed. Postpartum, the disease relapsed in 23/30 patients after a mean of 9.56 weeks. Therefore, these infants were exposed to anti-rheumatic drugs during lactation (anti-inflammatory drugs, Sulphasalazine and Hydroxicloroquine). No biological therapy was administrated during breastfeeding.

Conclusion. The data obtained regarding the relapse risk and breastfeeding while using antirheumatic drugs are generally within international recommendations. We need more experience to be able to manage difficult situations and to take the best decision for our female patients with RA.
\end{abstract}

Keywords: rheumatoid arthritis, breastfeeding, anti-rheumatic therapy

\section{INTRODUCTION}

Rheumatoid arthritis (RA) is a rheumatic condition that may affect individuals of all ages, therefore of fertile age as well and it is more frequently associated with the female gender.

RA is not a contraindication to pregnancy and breastfeeding; however, several recommendations must be considered, both before conception and during pregnancy and lactation.

If during pregnancy patients spontaneously have a good disease control, however, it is rather frequent that postpartum the disease relapses. Nevertheless, even in such circumstances, there are therapeutic solutions which are compatible with breastfeeding (1). The main purpose of this analysis is to evaluate the pregnancy outcomes of Romanian female patients with RA and, in the postpartum period, the risk of disease flare, the exposure to anti-rheumatic medication and the adherence to breastfeeding.

\section{PATIENTS AND METHODS}

The study was carried out between 2012 and 2016, with the participation of several Romanian centers of rheumatology: Dr Ion Cantacuzino Clinical Hospital, Colentina Clinical Hospital, and "Ana Aslan" National Institute of Gerontology, Bucharest; Rehabilitation Clinical Hospital, Cluj Napoca; Rehabilitation Clinical Hospital, Iasi; Ploiesti City Hospital, Ploiesti; "Sf. Apostol Andrei” Galati Emergency Hospital, Galati; CF Craiova Clinical Hospital, Craiova. It is a pilot study, strictly observational, non-interventional and included a prospective as

Correspondence address:

Anca Bobirca, Carol Davila University of Medicine and Pharmacy, 37 Dionisie Lupu Street, Bucharest

E-mail: carmenmarinamihai@yahoo.com 
well as a retrospective part. Patients with RA according to the 1980 American College of Rheumatology classification criteria, who had been diagnosed with RA before conception and who had at least one pregnancy after the diagnosis of RA were included. We recorded the pregnancy outcome, the occurrence of disease relapse postpartum and the exposure to antirheumatic medication during lactation. The study has been approved by the ethics committee of the Dr. Ion Cantacuzino Clinical Hospital and patients were included after informed consent.

\section{RESULTS}

Forty-one female patients with RA were included, all having at least one pregnancy after the diagnosis of RA. There were 72 pregnancies, of which more than half $(54.16 \%)$ have been planned, with an average age at the time of conception of 31.8 years, range 22-42 years. The pregnancy outcome for the entire group is illustrated in Fig. 1.

The mean duration of pregnancies in patients who gave birth has been of 35.37 weeks, ranging between 30 and 40 weeks. There were 3 pregnancies with twins. Three births occurred before week 37 .

The 42 newborns have had a mean weight of $2,715 \mathrm{~g}$, therefore within the normal range as specified by international recommendations.

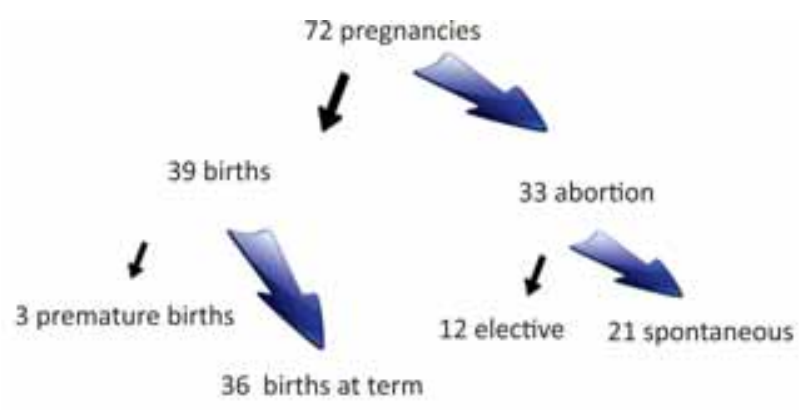

FIGURE 1. Outcome of pregnancy in 72 patients with rheumatoid arthritis

It is quite well known that RA may flare postpartum. Only 7 cases from all patients who gave birth didn't experience postpartum flares. In the other 23 patients, the mean time to flare was 9.56 weeks since parturition, range 1-24 weeks. Therefore, at approximately 10 weeks, the arthritis has relapsed in these patients as expected, an event associated with higher disease activity before conception and intrapartum. Time to relapse was shorter in patients with high disease activity during the pregnancy.

Of the 39 mothers, 30 were able to breastfeed, with a mean breastfeeding period was 23.76 weeks, range 4-108 weeks. Exposure to antirheumatic medication during breastfeeding occurred in a small part of patients, as most patients were afraid of associating medication during lactation. When the disease relapsed, the majority chose to discontinue breastfeeding, in order to restart therapy. No adverse events attributed to maternal medication were recorded in infants. The exposure occurred firstly to non-steroidal anti-inflammatory medication (NSAIDs) and glucocorticoids (GC) and secondly to the Sulphasalazine (SSZ) and Hydroxychloroquine (HCQ), as early as the $4^{\text {th }}$ week of breastfeeding. There were neither recorded exposures to Methotrexate (MTX) or Leflunomide (LFN), nor to biological therapies. Patients suffering from very active and difficult to control RA were recommended to interrupt breastfeeding in favor of resuming more potent synthetic medication.

\section{DISCUSSION}

Our study shows a considerably high rate of adverse pregnancy outcomes in patients with RA, mostly by pregnancy loss (21 spontaneous abortions in 72 pregnancies) and more rarely by prematurity ( 3 out of 72 patients). As expected, the disease flared postpartum, with a rather short time to flare, requiring anti-rheumatic medication. Most patients succeeded to breastfeed, and anti-rheumatic medication administered during breastfeeding was restricted to low-risk agents such as NSAIDs, GC, SSZ and HCQ. No patient reported the use of high-risk medicatin such as MTX or LFN during lactation.

Limitations of our study come from the low number of patients with pregnancies after the diagnosis of RA, the heterogeneity of the group and the various therapeutic strategies during pregnancy in the different rheumatology centers participating in this study. In the last part of this paper, we present an overview of the postpartum evolution of RA and the therapeutic recommendations regarding this period.

\section{Why does RA relapse postpartum?}

Rheumatoid arthritis frequently flares during the first 3 to 4 months postpartum. Recent prospective surveys have shown a lower rate of the postpartum flares, relapses being associated mostly with an insufficient management of RA during pregnancy and postpartum. The decrease of postpartum flares during the last decades might be explained by early initiation or re-initiation of anti-rheumatic medication postpartum $(2,3)$. 
A Switzerland workgroup has shown that the postpartum relapse rate correlates with the disease activity before conception, therefore the more stable the disease (remission or low activity) before pregnancy, the lower the risk of reactivation intrapartum or postpartum will be (4).

Another study found a higher postpartum relapse risk in women with RA who were breastfeeding for the first time, however, this supposition could not be verified by other studies $(3,5)$.

RA disease activity improves during pregnancy under the influence of the hormonal level and immune system changes, so postpartum RA reactivation might be due to the return to the hormonal and humoral systems' status to their preconception stage (6).

Another theory explains the RA postpartum flare risk by the high prolactin level in patients who are breastfeeding, since prolactin is a hormone known for its pro-inflammatory role by increasing the B lymphocytes proliferation, enhancing the development of antigen presenter cells, increasing the production of immunoglobulins and up-regulation of pro-inflammatory interleukins (IL) such as IL-1, IL-6, and IL-12 (7).

At the same time, the association of prolactin with RA relapse may be confounded by RA treatment, as breastfeeding RA patients use less antirheumatic medication (therefore the disease may be insufficiently controlled), while patients who aren't breastfeeding are usually receiving more medication, therefore obtaining a better postpartum therapeutic control of RA $(3,6)$.

The onset of RA postpartum is not rare and it seems to be influenced by the same immune-hormonal regulating mechanism; it is probable that pregnancy itself is responsible for postponing the onset of the disease by means of its tolerance mechanisms towards fetal antigens (8).

While the fact that RA is reactivated postpartum is recognized since a long time, the novelty lies in better disease control, usually achieved by continuing anti-rheumatic drugs compatible with lactation. Improving our knowledge in this field helps us reach a better balance between breastfeeding, the control of the disease and the evolution of the infant.

\section{Breastfeeding}

Breastfeeding is beneficial both for the infant and the mother; sometimes, however, the disease cannot be controlled by medication allowed during lactation and the solution is to cease breastfeeding, in favor of the mother and disadvantaging the infant.
Human breast milk contains specific amounts lactose, oligosaccharides, fat, proteins (including type A immunoglobulin A, IgA) and minerals. Medications administered to the mother occur in milk mostly by diffusion; therefore only fractions of the respective medication are transferred into milk. Large-sized proteins, heavy weight- or ionized molecules, as well as non-lipid medications will not succeed in passing into maternal milk.

A drug's concentration in maternal serum and its half time influence proportionally its penetration into breast milk. Once the child is exposed to the medication by breastfeeding, a special warning must be kept if the fetal serum concentration exceeds $10 \%$ of the infantile therapeutic dosage (8).

Another variable influencing medication transfer into breast milk is the time elapsed from its ingestion to breastfeeding, as most medications reach a serum height at approximately 1 or 2 hours after ingestion; therefore, the exposure of the infant to medication can be decreased by postponing the breastfeeding moment.

Fetal serum concentration of the medicine also depends on fetal intestinal absorption. Newborns prematures are thus more exposed to adverse events to medication administered to the breastfeeding mother, because their gastrointestinal tract is still immature and allows medication to pass into the infant's blood. Moreover, medication clearance is decreased due to the immaturity of the fetal metabolic pathways (1).

Different from the intrapartum period, during breastfeeding, the infant's exposure to risky medication does not happen incidentally, but by the decision of the mother and of the rheumatologist, in collaboration with the neonatologist or pediatrician.

Generally, anti-rheumatic medication has comparable intrapartum and postpartum precautions; in Table 1, the medication used during lactation is grouped according to risks.

TABLE 1. Anti-rheumatic medication and lactation

\begin{tabular}{|c|c|}
\hline \multicolumn{2}{|c|}{ Anti-rheumatic medication and breastfeeding } \\
\hline Low Risk & $\begin{array}{l}\text { Nonsteroidal anti-inflammatories (NSAIDs) } \\
\text { Glucocorticoids (GC) } \\
\text { Hidroxicloroquine (HCQ) } \\
\text { Sulphasalazine (SSZ), } \\
\text { TNF- } \alpha \text { blockers (Anti TNF- } \alpha \text { ) } \\
\text { Cyclosporine (CYC) } \\
\text { Azathioprine (AZA) }\end{array}$ \\
\hline High Risk & $\begin{array}{l}\text { Methotrexate (MTX) } \\
\text { Leflunomide (LFN) }\end{array}$ \\
\hline Unknown Risk & $\begin{array}{l}\text { Anakinra, Rituximab, } \\
\text { Abatacept, Tocilizumab, Tofacitinib }\end{array}$ \\
\hline
\end{tabular}


NSAIDs are accepted during breastfeeding, they transfer into breast milk in a small degree. It is recommended to use NSAIDs with a short half-life, in the smallest efficient doses, the most recommended being ibuprofen (8).

A special precaution should be given in newborns with icterus, where NSAIDs may favor the development of nuclear icterus (9).

By experts' opinions, non-selective NSAIDs are compatible with breastfeeding, while regarding COX2-selective NSAIDs there is enough data only for Celecoxib, which is accepted during breastfeeding, while the rest of the COX2-selective NSAIDs are contraindicated (10).

\section{Glucocorticoids}

Prednisone transfers into breast milk in a slightly higher proportion than NSAIDs (10\% compared to $5 \%$ ). The recommendation for Prednisone is to limit the dosage to less than $20 \mathrm{mg}$, as well as to postpone the breastfeeding moment by at least 4 hours since medication intake. Doses higher than $50 \mathrm{mg}$ per day require precautions and monitoring of the infantile suprarenal gland (10).

\section{Synthetic DMARDs and breastfeeding}

Sulphasalazine is composed of mesalazine and sulphapyridine. Mesalazine (5- aminosalicylic acid) does not transfer to breast milk or does so in a very low degree, while sulphapyridine is excreted into milk and the infantile serum concentration may reach $10 \%$ of the sulphapyridine concentration in the mother's serum. Sulphasalazine is allowed during breastfeeding, although rarely it has induced diarrhea in the infant and exceptionally, sanguinolent diarrhea (one case described in the literature).

A special precaution regards prematures, especially those with glucose-6-phosphodiesterase (G6PD) deficit and those with hyperbilirubinemia, in all of whom the risk of developing nuclear icterus is high when exposed to SSZ (10).

Hydroxychloroquine transfers into breast milk, but the fetal dose is much lower than the infantile therapeutic dose. The experts agree $100 \%$ to the continuation of breastfeeding during this treatment (10).

Azathioprine trasnfers into breast milk, but the infant serum concentration reached by breastfeeding is much smaller than the pediatric concentration recommended in transplant. Azathioprine is compatible with breastfeeding; however, a special attention is kept in the context of a deficit of thiopurine-methyltransferase (10).

Methotrexate is not recommended during breastfeeding. It is excreted into breast milk to a low degree, due to its insoluble lipidic form at the physiological pH. However, it is incompatible with breastfeeding because it accumulates in the tissues of the newborn and may lead to immunosuppression, neutropenia, development deficits and carcinogenesis $(10,11)$.

Leflunomide is not recommended during breastfeeding, although there are no data regarding its excretion into breast milk (12).

Cyclophosphamide is an alkylating agent that transfers into breast milk and onto the infant, where it can cause neutropenia and medullary suppression, therefore it is contraindicated during lactation (10).

\section{Biologic Agents and Breastfeeding}

Most anti TNF- $\alpha$ agents are monoclonal antibodies of a subclass of IgG. Therefore, their secretion into breast milk is limited. Also, all anti-TNF- $\alpha$ agents are large protein molecules that get split up by the enzymes of the fetal digestive tract, therefore we encounter a double limitation of the fetal biological availability $(10,13)$.

For instance, for children exposed in utero (last trimester) to Etanercept, this agent could be detected in the fetal blood immediately postpartum; however, when Etanercept was administered to the mother during breastfeeding, no drug could be identified in the infant's serum (10).

Infliximab in breast milk and in the serum of the infants who were breastfed has levels below the detection limit, even when the mother has received Infliximab as far as week 30 of pregnancy and has resumed taking it immediately postpartum, although the mother serum was positive for Infliximab (14).

The PIANO study, a prospective study that analyzed 102 patients exposed to biological therapy on the entire duration of the pregnancy, has shown that among children who were breastfed $(72 \%$ of the group), the rate of infections has not been higher, although in some cases concentrations of Adalimumab or Infliximab were detected in the mother's milk (13).

In the context of the exposure over the duration of the entire pregnancy and breastfeeding to Certolizumab pegol, it could not be identified in the mother's milk (15).

Experts in Gastroenterology and Rheumatology accept the continuation of breastfeeding while under 
anti-TNF- $\alpha$ therapy. However, in Romania this has not been specified yet in the treatment protocols.

Except for TNF- $\alpha$ blockers, for the other biological therapies such as Rituximab, Anakinra, Tocilizumab and Abatacept, there are no data regarding breastfeeding and therefore these are contraindicated during this period. However, given the pharmacokinetic characteristics of these medications, lactation should not be totally discouraged if other therapeutic options are missing (10).

\section{CONCLUSIONS}

Romanian RA patients are treated according to the EULAR recommendations, also in regard to this important chapter in their lives: pregnancy and lactation. It is important to discuss this aspect with young patients, beginning from the moment of the diagnosis of RA: to choose together the anti-rheumatic medication, to effectively plan a pregnancy and to be able to manage the disease before conception, intrapartum, postpartum and during breastfeeding, having a good cooperation with the attending gynecologist and neonatologist.

\section{Aknowledgement}

The study has been made possible with the help of our collegues: Mariana Sasu, Claudia Ciofu, Liviu Macovei, Andrei Martin (Dr Ion Cantacuzino Hospital, Bucharest,); Victoria Jugravu (Ana Aslan National Institute of Gerontology, Bucharest); Mihaela Micu (Rehabilitation Clinical Hospital, Cluj Napoca); Codrina Ancuta (Rehabilitation Clinical Hospital, Iasi); Magda Parvu, Livia Stanescu-Rautzoiu (Colentina Clinical Hospital, Bucharest); Carmen Dumitru, Mihaela Agache (Ploiesti City Hospital, Ploiesti); Mariana Pavel ("Sf. Apostol Andrei" Galati Emergency Hospital, Galati); and Anca Musetescu (CF Craiova Clinical Hospital, Craiova).

\section{REFERENCES}

1. Sammaritano L.R., Bermas B.L. Rheumatoid arthritis medications and lactation. Curr Opin Rheumatol. 2014 May; 26(3):354-60. doi: 10.1097/BOR.0000000000000055.

2. Man Y.A., Dolhain R.J., van de Geijn F.E., Willemsen S.P., Hazes J.M. Disease activity of rheumatoid arthritis during pregnancy: results from a nationwide prospective study. Arthritis Rheum. 2008; 59:1241-8.

3. Barrett J.H., Brennan P., Fiddler M., Silman A. Breastfeeding and postpartum relapse in women with rheumatoid and inflammatory arthritis. Arthritis Rheum. 2000; 43:1010-5.

4. Forger F., Vallbracht I., Helmke K., Villiger P.M., Ostensen M. Pregnancy mediated improvement of rheumatoid arthritis. Swiss Med Wkly. 2012; 142:w13644

5. Nelson J.L., Ostensen M. Pregnancy and rheumatoid arthritis Rheum Dis Clin North Am. 1997; 23:195-212.

6. Dolhain R.J. Rheumatoid arthritis and pregnancy; not only for rheumatologists interested in female health issues. Ann Rheum Dis 2010; 69:317-318.

7. Orbach H., Shoenfeld Y. Hyperprolactinemia and autoimmune diseases. Autoimmun Rev 2007; 6:537-542.

8. Gayed M., Gordon C. Rheumatology Pregnancy and Rheumatic Diseases. 2007; 46(11):1634-1640.

9. Niebyl J.R., Simpson J.L. In: Gabbe SG, Nielbly JR,Simpson JL, Landon MB, Galan HL, Jauniaux ERM, Driscoll DA. Drugs and environmental agents in pregnancy and lactation: embryology,

teratology, epidemiology, Obstetrics: normal and problem pregnancies, 6th ed. Philadelphia, PA: Saunders (Elsevier); 2012.

10. The EULAR points to consider for use of antirheumatic drugs before pregnancy, and during pregnancy and lactation. Ann Rheum Dis. 2016 May; 75(5):795-810. doi: 10.1136/ annrheumdis-2015-208840. Epub 2016 Feb 17.

11. Namieta M. Janssen, Marcia S. Genta, The Effects of Immunosuppressive and Anti-inflammatory Medications on Fertility, Pregnancy, and Lactation, Arch Intern Med. 2000; 160(5):610-61

12. $B S R$ and $B H P R$ guideline on prescribing drugs in pregnancy and breastfeeding-Part I: standard and biologic disease modifying antirheumatic drugs and corticosteroids. Rheumatology (Oxford). 2016 Sep; 55(9):1693-7. doi: 10.1093/rheumatology/kev404. Epub 2016 Jan 10.

13. Gisbert J.P., Chaparro M. Safety of anti-TNF agents during pregnancy and breastfeeding in women with inflammatory bowel disease. Am J Gastroenterol 2013; 108:1426-38.

14. Zelinkova Z., de Haar C., de Ridder L., et al. High intra-uterine exposure to infliximab following maternal anti-TNF treatment during pregnancy. Aliment Pharmacol Ther 2011; 33:1053-8.

15. Mahadevan U., Wolf D.C., Dubinsky M., et al. Placental transfer of anti-tumor necrosisfactor agents in pregnant patients with inflammatory bowel disease. Clin Gastroenterol Hepatol 2013; 11:286-92 [quiz: e24]. 